\title{
LA FORMACIÓN DE HABILIDADES EXPERIMENTALES DE FÍSICA EN LOS ESTUDIANTES DE CARRERAS NO PEDAGÓGICAS
}

\section{FORMATION OF EXPERIMENTAL SKILLS IN PHYSICS ON STUDENTS FROM NON-PEDAGOCICAL CAREERS}

Juan Carlos Martin Llano. MSc.

martin@upr.edu.cu

Luis Enrique Hernández Amaro. Dr. C.

\section{RESUMEN}

En el presente artículo se muestra la evolución histórica del proceso de formación de las habilidades experimentales de la Física en las carreras universitarias y la sistematización de las tendencias del Proceso de formación de las habilidades experimentales de la Física, en los estudiantes universitarios de carreras no pedagógicas.

Palabras claves: habilidades experimentales, tendencias, formación

\section{ABSTRACT}

This paper shows the historical evolution of the experimental ability formation process of Physics at undergraduate studies and the systematization of trends in the formation process of experimental skills in Physics on university students from non-pedagogical careers.

Keywords: experimental skills, trends, formation

Recibido: enero de 2016 Aprobado: abril de 2016

\section{Introducción}

La formación de profesionales, en la actualidad, se desarrolla en un contexto de alto desarrollo tecnológico y en condiciones de un mundo globalizado. La misión de la universidad consiste en preservar, desarrollar y promover la cultura de la humanidad en plena integración con la sociedad. Llegar con ella a todo el pueblo contribuyendo al desarrollo sostenible, con el paradigma: La universidad para todos, durante toda la vida.'

La preocupación constante por el mejoramiento de la educación superior ha dado lugar a que se apliquen varios planes denominados $\mathrm{A}, \mathrm{B}, \mathrm{C}, \mathrm{C}$ modificado y $\mathrm{D}$, además se dotó de recursos técnicos y nuevos equipamientos de laboratorios en las diferentes enseñanzas, en las cuales se insertan diferentes ciencias experimentales como la Física.

La Física, en la carrera de Ingeniería Agronómica, constituye una disciplina básica estructurada en dos asignaturas: Física I y II. En el proceso de enseñanza aprendizaje de la misma, la formación de las habilidades experimentales constituye un aspecto trascendente, necesarias para la ejecución de la actividad experimental y la formación de actitudes sociocientíficas y socioprofesionales en los Ingenieros Agrónomos en formación.

En los planes de estudio de la Física para la carrera de Ingeniería Agronómica, se dedica un fondo de tiempo a la ejecución de actividades prácticas. Dentro de las actividades prácticas se encuentran: clases prácticas, seminarios y laboratorios de acuerdo a la tipología de clases que aparecen reglamentadas.ii

La problemática relacionada con el trabajo experimental de Física, cuenta con una tradición en la investigación pedagógica (Fuentes 1989, Herrera, 1982, 1985, 1989, 1995; Leyva, 2002; Colado, 2003; Moltó, 2004; Fundora, 2008; Pozo, 2011, 2013, Morales, 2006, 2014, entre otros). En dichas investigaciones, se ha demostrado la importancia de la formación de conocimientos y habilidades, con énfasis 
en la actividad experimental. Todo ello resalta que el experimento constituye una vía para realizar la vinculación de la teoría con la práctica durante la enseñanza, en la cual los conocimientos se transforman en convicciones.

Las vivencias acumuladas por los autores en la dirección del proceso de enseñanza aprendizaje de la Física, les ha permitido apreciar por un lado, la realidad de las limitaciones del proceso de formación de habilidades experimentales de Física en los estudiantes de la carrera de Ingeniería Agronómica, al desarrollarse de forma asistemática, desintegrado y con poca profesionalización, sin que responda a la lógica de la profesión de Ingeniero Agrónomo y por el otro, la necesidad de potenciar didácticamente el proceso de formación de habilidades experimentales de Física de modo integrado, sistémico desde la lógica de la profesión del Ingeniero Agrónomo con enfoque investigativo; sobre este aspecto se debate en el presente artículo.

\section{Desarrollo}

A continuación se muestra la evolución histórica y lógica del proceso de formación de las habilidades experimentales de la Física en las carreras universitarias, para con posterioridad mediante la sistematización de los referentes teóricos, se identifican las regularidades del Proceso de Formación de las Habilidades Experimentales de la Física (PFHEF) en los estudiantes universitarios.

A partir del estudio histórico-lógico realizado al proceso de formación de habilidades experimentales en la enseñanza-aprendizaje de la Física y utilizando como criterios: la vía del conocimiento científico empleado y el tipo de enfoque en el proceso de enseñanza aprendizaje, a partir de estos dos criterios se identifican a continuación las tendencias en el proceso de formación de habilidades experimentales en la enseñanza-aprendizaje de la Física. Se entiende como tendencia lo expresado por la Dra. C. María Elena Sánchez Toledo a la "Dirección u orientación probable que asume este en su desarrollo, resultante de la conjugación de las condiciones, factores, leyes externas e internas asociadas a este fenómeno." "iii

La enseñanza-aprendizaje de las ciencias experimentales y en particular la Física, se encuentra muy ligada a la propia evolución de la enseñanza aprendizaje a lo largo de la historia a nivel mundial, en el que se configuran una sucesión de tendencias, entre las que se destacan las siguientes:

Desde fines del siglo XVIII fue muy generalizada la tendencia a la enseñanza aprendizaje por transmisión-recepción de conocimientos, su representante John Loocke «se concibe al alumno como "página en blanco", los conocimientos se transmiten ya elaborados, mediante la conferencia magistral y la memorización como modelo de acción $»^{i v}$. El proceso de enseñanza aprendizaje de las disciplinas de ciencias está basado en una secuencia de conocimientos científicos definitivos, de verdades incuestionables.

En 1886, el educador cubano Enrique José Varona planteó: "se conforma un programa, para el programa se confecciona un texto, el profesor se esclaviza al texto y el alumno sabe que todo lo que tiene que hacer es aprenderse de memoria las respuestas a un grupo de preguntas y resolver determinados problemas."v

En el caso de la actividad experimental es esencialmente mediante demostraciones y el profesor le ofrece al alumno las conclusiones del mismo o como prácticas de laboratorio tipo receta. Aunque es la tendencia más antigua, todavía prevalece hoy en muchos países y niveles de educación por ser la más cómoda para el educador y económica para las instituciones educativas.

A lo largo de la historia mostró su poca eficiencia. El estudiante es pasivo, limitando su razonamiento, creatividad y desarrollo personal en su formación integral.

A mediados del siglo XIX, se propone poner en contacto a los estudiantes con el mundo que le rodea, al surgir la enseñanza de las ciencias por descubrimiento (Brunner 1960), que supone redescubrir lo ya descubierto (Gil, 1991). Esta tendencia influye en la década de los años 60 al 80 del siglo pasado en los movimientos educativos anglosajones.

En la misma se concibe el aprendizaje similar a las formas de trabajar de los científicos, son sustituidos los conocimientos como protagonista por los procesos. Tuvo repercusión en los proyectos como: el Physical Science Study Committee(PSSC), y el Biological Science Study Committe(BSSC) en Estados Unidos y el proyecto Nuffield en Inglaterra, su influencia en Cuba fue en cursos introductorios de la Educación Superior.

Del análisis realizado sobre la tendencia expuesta anteriormente se reconoce que posee ventajas como: el profesor, su rol es coordinar actividades experimentales, proporciona oportunidades de investigar que ayudan al desarrollo de habilidades de investigación, las interacciones son además de profesor-estudiante, también es estudiante-estudiante, relaciona al estudiante con el descubri- 
miento científico, utiliza métodos educativos menos autoritarios, los educandos se motivan más por aprender, por ser ellos los que «descubren las cosas».

Sus desventajas como: los fundamentos empírico - inductivo, plantea que el experimento es la fuente fundamental del conocimiento científico y que toda experiencia debe comenzar con la observación que es equivalente a percepción.

La tendencia pierde importancia infringiendo en enfoques simplistas, muy alejadas de como en realidad se producen los conocimientos científicos, se aprecia que no hay una elaboración teórica previa aspecto que limita la formación de habilidades experimentales, aunque se fue avanzando con respecto a la anterior.

A finales de 1970, surge la enseñanza integrada de las ciencias, como una tendencia establecida, con una orientación menos parcializada, más global de los conocimientos científicos. Según Jardinot Mustelier, Luis Roberto (2005) señala que si se pretende conectar con los intereses de los estudiantes y partir de los problemas de su entorno, hay que tener en cuenta que la percepción de esos problemas sea globalizadora, ya que no entiende de divisiones entre asignaturas. Mena Lorenzo (2010), refiere que se necesitaba organizar las materias en unos conceptos generales capaces de vencer las fronteras impuestas por la concepción disciplinar.

Desde este análisis se considera que en la actividad experimental desarrollada en la década de los 70', no se pudo valorar su concreción en la práctica pedagógica mediante acciones específicas que permitan una apropiación sólida de habilidades.

En la década de los 80's un relativo consenso (Driver Guesne,y Tiberghien 1989, Obone y Freyberg1991, Gil 1993) suscita el modelo constructivista (su exponente Piaget. 1940), en EE.UU y Europa. Este concibe el aprendizaje de las ciencias como una construcción del conocimiento, así la formación de las habilidades, parte de un conocimiento previo, y construcción personal del conocimiento. Se caracteriza por un aprendizaje relacionado con lo que ya sabe. Como aspecto significativo la caracteriza la gran flexibilidad en espacios, horarios, materiales y contenidos.

Se destaca en este periodo, el aprendizaje significativo Ausubel (1993). La mencionada tendencia se desarrolló en contraposición al aprendizaje por descubrimiento y tuvo sus principales exponentes en Ausubel (1960), Novak y Reigeluth (1970), se resalta al profesor como guía del aprendizaje del alumno.

La misma se dirigió a la jerarquía de conceptos a introducir y la elaboración de mapas conceptuales, para presentar de forma ordenada los conocimientos de modo que pudieran integrarse significativamente, es decir de forma intencionada en las estructuras conceptuales de los alumnos, de acuerdo con (Pozo, J.I), citado por Addine $(2007)^{v i}$, favorece el desarrollo de habilidades para el procesamiento de información y eleva la autoestima.

Según Gil (2008) en la antigua URSS se desarrollaron dos tendencias fundamentales que influyeron notablemente en Cuba después del triunfo de la Revolución y hasta mediados de la década del 90.

La primera se desarrolló en el proceso de enseñanza aprendizaje de la Física, fundamentalmente a partir de los trabajos de Razumovski, ${ }^{\text {vi }}$ denominada así, siguiendo el ciclo del conocimiento científico, la que debía transcurrir como se originaba el conocimiento en la ciencia. Como la concebía Descartes como el ir de lo general a lo particular. Esta tendencia tenía un enfoque empirista-inductivista.

La segunda tendencia desarrollada en la antigua URSS en las décadas del 70' y el 80' a partir de los trabajos de Majmutoviii (1979) fue la enseñanza problemática cuyos exponentes son Krulicy Rutnick. Se basa en que el proceso de enseñanza aprendizaje de las ciencias que debía partir de situaciones problemáticas o que creen conflictos en el educando y que lo motiven a actuar. Posee dos variantes: la exposición problemática que plantea situaciones contradictorias que conlleve a formularse hipótesis y la conversación heurística a partir de una situación problémica y la obtención del conocimiento a través de preguntas planteadas por el educador.

Se aprecia en estas tendencias un enfoque empirista inductivista, aún se limita el rol activo del estudiante como investigador. Existía un problema esencial, el no tener presente los conocimientos previos, por lo general, no sabía o lo que sabía eran conocimientos alternativos al científico y eran los que perduraban pasado un tiempo en que se olvidaban las cadenas verbales aprendidas. No concibió al estudiante en su integridad. En la enseñanza problémica, el educador tenía el papel más activo y la evaluación, lo que medía, era una actuación reproductiva, lo que no siempre se tiene en cuenta el nivel aplicativo y creativo, lo que repercute en la formación de habilidades y 
dentro de ellas, las experimentales.

Esta tendencia sistematiza conceptos aprendidos por los educando y su potencial teórico para enfrentar las diferentes acciones prácticas necesarias como las actividades experimentales, desde esta tendencia el autor considera que con un carácter más general, desde la última década del pasado siglo, surge la enseñanza desarrolladora (2001), dirigida al desarrollo y la socialización de los saberes desde los niveles de ayuda que el trabajo en grupo ofrece al desarrollo de los aprendizajes.

Desde la última década se hace énfasis en la enseñanza desarrolladora de las ciencias. Pedagogos reconocidos como Martínez, LI, M. (1986), Rico Montero, P. (2000), Castellanos, Doris (1999), Rodríguez-Mena, (2000), entre otros. La misma está basada en la pedagogía socialista fundamentada en el enfoque histórico-cultural de (Vygotsky. 1950), que considera que la enseñanza debe conducir al desarrollo integral de la personalidad de los estudiantes. Esta tiene lugar por medio de los sistemas actividad-comunicación, en los que estos se involucran, mediado por todos los sujetos con los que interactúan.

Desde el plano didáctico se considera que el desarrollo de los estudiantes puede ser dirigido mediante la utilización creadora de los componentes didácticos con carácter desarrollador y formativo. Para (Rodríguez-Mena, 1999), la enseñanza desarrolladora debe atender, aspectos como: el sujeto que aprende y el contexto. Igualmente debe tener muy preciso qué se aprende: los objetivos, contenidos y cómo se ejecutan los métodos y la evaluación.

En esta tendencia, soportada en los criterios de Majmutov (1983) asociada con el enfoque de problemas, presta atención al enfoque problémico que propicia las ciencias, lo que contribuye al desarrollo de la creatividad, formación de convicciones, sentimientos, actitudes propias de la actividad científica (Majmutov, 1983; Martínez, 1986) que ha hecho énfasis en motivar al alumno para la asimilación de los contenidos escolares desde la presentación de un problema a resolver.

Descubrir y formular problemas es una vía excelente y básica para movilizar la reflexión de los niños y la exploración de alternativas de solución creadoras; pero el problema no puede ser dado, impuesto desde afuera, sino elaborado por el propio sujeto o entre los sujetos que aprenden. (Rodríguez-Mena, 2000)ix.

La enseñanza desarrolladora, parte del desarro- llo alcanzado por el estudiante y del diálogo de la actividad colectiva, promoviendo el máximo desarrollo posible de sus potencialidades. Castellanos, Doris (1999)x plantea que "la educación desarrolladora promueve y potencia aprendizajes desarrolladores."

Las situaciones de aprendizaje desarrolladoras se caracterizan por su carácter consciente, reflexivo, problematizador, significativo y contextualizado. La organización de la metodología de trabajo en torno a lo problémico, lo heurístico, lo investigativo, lo creador, deben contribuir al desarrollo de las habilidades y capacidades implicadas en una actividad intelectual productiva, creadora y reflexiva.

El empleo de procedimientos analógicos y vivenciales, el uso del error y de la interrogación como estrategias didácticas, la enseñanza multisensorial, la simulación, el apoyo en la realización de proyectos, entre otros, constituyen procedimientos citados por (de la Torre, 1995) xi que contribuyen al desarrollo de una enseñanza activa, motivadora, dinámica y por ende, a la proyección de situaciones de aprendizaje desarrolladoras.

En la enseñanza-aprendizaje se han puesto de manifiesto otras aplicaciones, procedimientos, métodos de acuerdo a las exigencias y condiciones de la revolución del conocimiento en las que se encuentra el Modelo Sudbury, "en la que argumentan que aprender es un proceso que tú haces, no que se te hace a ti; que eso es cierto para todos y básico."xii

Se manifiesta en la enseñanza -aprendizaje el Modelo Proyectivo. Se basa en el aprendizaje a través de la formulación de proyectos, donde el docente propone un pretexto que es un elemento de interés para los interactuantes en el desarrollo de la propuesta.

El Modelo Proyectivo, xiii según el estudio realizado, plantea actividades con el propósito de que se preparen para la vida. Se trata de que los alumnos organizados por los maestros, encuentren una necesidad, planteen un proyecto de solución, recaben información, trabajen en la generación de soluciones, las pongan en marcha y evalúen al final. Es una imitación de lo que ocurre en la vida real, y en lo posible se apunta a intervenciones reales con consecuencias reales.

El modelo de proyecto incentiva la investigación en todas las líneas de conocimiento y desarrolla las potencialidades de los estudiantes. Se basa en el interés de cada uno de ellos, permite la construcción colectiva de normas, como una fuente importante de convivencia ciudadana. Plantea 
el respeto por el bien colectivo y construye el conocimiento a través de la experiencia.

Este modelo expresa algunas de sus características relevantes en el análisis tendencial del proceso de enseñanza - aprendizaje de la Física. Este favorece el carácter activo del estudiante en el proceso de formación de las habilidades experimentales para la apropiación de acciones teóricas y prácticas. El desarrollo de las diferentes actividades de carácter experimental juega su rol en el "saber hacer" y transferir el saber hacer a otros contextos, se aprecian irregularidades de la introducción progresiva de la actividad experimental.

\section{Conclusiones}

A partir del estudio de las tendencias fundamentales del proceso de formación de habilidades experimentales en la enseñanza-aprendizaje de la Física, surge la necesidad de indagar soluciones en apoyo a la actividad experimental durante la enseñanza de la Física.

En la dirección del proceso de enseñanza aprendizaje de la Física, se aprecian las limitaciones del proceso de formación de habilidades experimentales en los estudiantes de la carrera de Ingeniería Agronómica, al desarrollarse de forma asistemática, desintegrado y con poca profesionalización, sin que responda a la lógica de esta profesión, además de la necesidad de potenciar didácticamente el proceso de formación de habilidades experimentales de Física de modo integrado, sistémico desde la lógica de la profesión del Ingeniero Agrónomo con enfoque investigativo.

En esencia, las tendencias en la FHEF se resumen en: 1- el desarrollo de habilidades experimentales de la Física desde el proceso de transmisión-recepción de conocimientos; 2- la enseñanza de las ciencias por descubrimiento, con métodos muy cercanos al de la investigación; 3- la enseñanza integrada de las ciencias que se concibe como una construcción del conocimiento y unido a ellos ocurre la formación de las habilidades; 4- la enseñanza problémica, que se valora en el desarrollo de las habilidades como un enfoque empirista inductivista; y, 5- el aprendizaje desarrollador, concepción que se inserta en la actualidad por significar la importancia del desarrollo de las habilidades experimentales en las ciencias exactas y naturales, donde la Física tiene un lugar especial.

\section{Referencias Bibliográficas}

1. Álvarez de Zayas, C. M. La Escuela en la vida. Editorial Pueblo y Educación. La Habana. (1992).

2. Bararabanchikov. A.V Cuestiones actuales de la enseñanza y la educación en los guardafrontera. Moscú. (1980)

3. Castellanos, D. La comprensión de los procesos del aprendizaje: apuntes para un marco conceptual. Centro de Estudios Educacionales, ISPEJV, La Habana. (1999):

4. Chukina G.IActivación de la actuación cognitiva de los alumnos.Moscú. (1979).

5. De la Torre, S. Estrategias de enseñanza aprendizaje creativos. En colectivo de autores. Pensar y crear: Estrategias, métodos y programas. La Habana: Editorial Academia. (1995).

6. López López, M. Sabes enseñar a describir, definir, argumentar. Editorial Pueblo y educación la Habana. (1990).

7. Maura, Gonzales. V. Psicología para Educadores. Ed: Pueblo y Educación. La Habana. (2001)

8. Petrovski, A.V. Psicología General. Moscú. (1980).

9. Petrovski, A.V. Psicología General. Editorial de libros para la Educación. Ministerio de Educación. La Habana. . (1979)

10. Rodríguez-Mena, M. "El enfoque Crítico-Reflexivo en Educación”. En Revista Educación. No 99, enero-abril del 2000. p. 8-11, La Habana. (2000):

11. Silva R. Acerca de los conceptos de Hábito y habilidad. imprenta central de las FAR .La Habana. (1986).

12. Surnichenko S.I Fundamentos de Psicología en el trabajo partidista. Moscú. (1978).

13. Talizina N. F. Conferencia sobre la Enseñanza en la educación Superior, Habana. (1984).

14. Tamayo, BSistema de habilidades experimentales de la disciplina Química Inorgánica para la Licenciatura en Educación, especialidad de Química. Tesis de Doctorado para la obtención del título de Doctor en Ciencias Pedagogía. Instituto superior pedagógico, Holguín, Cuba. . (2000).

15. Pozo, E; Valcárcel, N, entre otros. La medición, atributo relevante en el Modelo didáctico metodológico para el trabajo experimental en las disciplinas de física para los estudiantes de la carrera de Licenciatura en Educación de la especialidad Matemática - Física de la UCP "Rafael María de Mendive".Revista IPLAC. Volumen No. 5, septiembre-octubre. 2011. En: www.revista.iplac. 
rimed.cu. ISSN: 1993-6850. La Habana. (2011).

16. Pozo, E; Valcárcel, $\mathrm{N}$, entre otros. Estrategia didáctica dirigida a la preparación para el trabajo experimental de los estudiantes de la carrera Licenciatura en Educación, especialidad de Matemática Física. CD-Rom Memorias del evento internacional Pedagogía '2013. ISBN, 978-95918-0881- 3. (2013).

17. Pozo, E; Valcárcel, N, entre otrosMedir una Habilidad Profesional específica para el profesor de Física. Revista Científica Pedagógica Mendive. Año 9 No 33 Octubre - Diciembre 2010. ISSN 1815 - 7696. . (2010).

18. Morales, H; Valcárcel, N, entre otros. Habilidades profesionales específicas para la enseñanza de la Física en la formación inicial de la especialidad de Matemática - Física en la UCP "Rafael María de Mendive". Revista Científica Mendive No.47 año 12 abril - junio con ISSN: 1815-7606. . (2013)

i http://www.ecured.cu/index.php/Universidad_cubana.

\section{Notas}

ii Álvarez C., Horruitiner P., Fuentes H.(1986) Tendencias en la Enseñanza de la Física para Ingenieros en Cuba, Rev. Cub. de Educ. Sup., Vol VI, No 1

iii Sánchez Toledo, ME (et al). El desarrollo de tendencias en el proceso docente educativo. En CD-Rom. Doctorado curricular en Ciencias Pedagógicas. UCPEJV. La Habana. 2005: p 60.

iv Jiménez M,P.(S/F).Didáctica de las ciencias experimentales, Modelos didácticos ED. Marfil S.A Alcoy España.

v E. Varona (1991).Trabajos de Educación y Enseñanza. Editorial Pueblo y Educación. La Habana.

vi Addine Fernández, F. y otros. (2007).Didáctica: teoría y práctica. Ed. Pueblo y Educación, La Habana,pág 92.

vii Razumovski, V. (1987). El Desarrollo de las Capacidades Creadoras en los Estudiantes. Editorial Pueblo y Educación. La Habana.

viii I. Majmutov (1979).Enseñanza Problemática. Editorial Pueblo y Educación. La Habana.

ix Rodríguez-Mena, M. (1999): Aprender con calidad (inédito). La Habana, CIPS.
Rodríguez-Mena, M. (2000): "El enfoque Crítico-Reflexivo en Educación”. En Revista Educación. No 99, enero-abril del 2000. p. 8-11, La Habana.

x Castellanos, Doris (1999): La comprensión de los procesos del aprendizaje: apuntes para un marco conceptual. Centro de Estudios Educacionales, ISPEJV, La Habana

xi De la Torre, Saturnino. (1995). Estrategias de enseñanza -aprendizaje creativos. En colectivo de autores. Pensar y crear: Estrategias, métodos y programas. La Habana: Editorial Academia.P.12.

xii Greenberg, D. (1987). The Sudbury Valley School ExperienceBacktoBasics.(Accedido, 18 de diciembre de 2014.)

xii Modelos de enseñanza.De Wikipedia, la enciclopedia libre 